\title{
Geographic variation in the prevalence of Epstein-Barr virus-positive diffuse large B-cell lymphoma of the elderly: a comparative analysis of a Mexican and a German population
}

Annegret Hofscheier ${ }^{1, *}$, Ana Ponciano,*, Irina Bonzheim ${ }^{1}$, Patrick Adam ${ }^{1}$, Carmen Lome-Maldonado ${ }^{2}$, Teresa Vela ${ }^{3}$, Evelyn Cortes ${ }^{4}$, Carlos Ortiz-Hidalgo ${ }^{5}$, Falko Fend $^{1}$ and Leticia Quintanilla-Martinez ${ }^{1}$

${ }^{1}$ Institute of Pathology, Eberhard-Karls-University of Tübingen, Tübingen, Germany; ${ }^{2}$ Department of Pathology, Instituto Nacional de Ciencias Medicas y Nutricion Salvador Zubiran, Mexico City, Mexico; ${ }^{3}$ Department of Pathology, Instituto Nacional de Cancerologia, Mexico City, Mexico; ${ }^{4}$ Department of Pathology, Hospital Juarez, Mexico City, Mexico and ${ }^{5}$ Department of Pathology, American British Cowdry Hospital, Mexico City, Mexico

\begin{abstract}
Epstein-Barr virus (EBV)-positive diffuse large B-cell lymphoma of the elderly was included as a provisional entity in the 2008 WHO lymphoma classification. Most reports of this disease come from Asia and little is known about it in other regions of the world, including Latin America. Therefore, in this study, 305 diffuse large B-cell lymphomas in patients above 50 years were analyzed, 136 from Mexico and 169 from Germany. EBV was detected by Epstein-Barr early RNA (EBER) in situ hybridization. Only cases with EBER + in the majority of tumor cells were regarded as EBV + diffuse large B-cell lymphoma. The prevalence of EBV + diffuse large B-cell lymphoma in Mexican patients was found to be $7 \%$ (9 of 136), whereas only $2 \%$ (4 of 169) of the German cases were positive. The median age at diagnosis was 66 years in the Mexican cohort, as opposed to 77 years in the German group. The site of presentation was in both groups predominantly nodal in nine cases $(70 \%)$ and extranodal in four cases $(30 \%)$. Of the $13 \mathrm{EBV}+$ cases, $10(77 \%)$ were classified as polymorphic and $3(23 \%)$ as monomorphic type. The polymorphic cases showed a non-germinal center B-cell immunophenotype (CD10 - MUM1 +). Twelve cases (92\%) were LMP1 positive and two (15\%) expressed EBNA2. An interesting finding was the high frequency of EBV type B with the LMP1 30 bp deletion found in the Mexican cases $(50 \%)$. Eight of the 11 evaluable cases were B-cell monoclonal by polymerase chain reaction. In summary, we found a similar prevalence of EBV + diffuse large B-cell lymphoma of the elderly in a Mexican population compared with what has been reported in Asian countries, and in contrast to the low frequency in Western populations (1-3\%). However, compared with the Asian series, the Mexican patients were younger at diagnosis, presented predominantly with nodal disease and rarely expressed EBNA2 protein.
\end{abstract}

Modern Pathology (2011) 24, 1046-1054; doi:10.1038/modpathol.2011.62; published online 15 April 2011

Keywords: EBV; elderly; large cell lymphoma; Mexico

Correspondence: Dr L Quintanilla-Martinez, MD, Institute of Pathology, University Hospital Tuebingen, Liebermeisterstrasse 8, Tuebingen 72076, Germany.

E-mail: leticia.quintanilla-fend@med.uni-tuebingen.de

* These authors contributed equally to this work.

Received 13 December 2010; revised 7 January 2011; accepted 9 January 2011; published online 15 April 2011
Epstein-Barr virus (EBV) is a ubiquitous herpesvirus that infects $>90 \%$ of the human population establishing persistent latent infection in the host. ${ }^{1}$ Although EBV infection is benign in most individuals, it has been linked to the etiology of a rather broad spectrum of B-cell lymphoproliferations both 
in the post-transplant and iatrogenic setting, ${ }^{2}$ as well as to malignant lymphomas in immunocompetent hosts such as Burkitt lymphoma, lymphomatoid granulomatosis and a proportion of classical Hodgkin lymphoma. ${ }^{3,4}$

In 2003, Oyama et $a l^{5}$ reported 22 cases of EBVassociated diffuse large B-cell lymphoma that occurred in elderly individuals ( $>60$ years) without apparent immune deficiency, and suggested a relationship with immunological deterioration as a result of the aging process. Further reports from Japan $^{6}$ and Korea $^{7}$ showed that EBV-associated diffuse large B-cell lymphoma accounts for $8-10 \%$ of diffuse large B-cell lymphoma in elderly Asian patients without a documented predisposing immunodeficiency. As a result of these reports, EBVpositive diffuse large B-cell lymphoma of the elderly was included in the revised 2008 WHO classification of tumors of hematopoietic and lymphoid tissues $^{8}$ as a 'provisional' category. It is defined as an EBV + clonal B-cell proliferation that occurs in patients older than 50 years and without any known immunodeficiency, immunosuppression, transplantation, autoimmune disease or prior lymphoma. Cases of other well-defined lymphoma entities that may be EBV positive are excluded from this provisional category. Morphologically, EBV + diffuse large B-cell lymphoma of the elderly often shows features of post-transplant lymphoproliferative disorders, although it develops in clinically immunocompetent patients. Helpful pathologic features that suggest EBV association include large transformed cells, polymorphic infiltrate, plasmacytic and plasmablastic morphology, Hodgkin and Reed-Sternberg-like cells, angioinvasion, necrosis and non-germinal center B-cell immunophenotype.

Interestingly, EBV-associated lymphomas like NK/T-cell lymphoma, ${ }^{9}$ T-cell lymphoproliferative disorders of childhood ${ }^{10}$ and EBV + classical Hodgkin lymphoma ${ }^{11,12}$ are more commonly observed in Asia and in Latin American countries suggesting an ethnic predisposition for the development of EBV-associated lymphoproliferative disorders. This also seems to be true for EBV + diffuse large B-cell lymphoma of the elderly, which has been reported to account for $8-10 \%$ of diffuse large B-cell lymphoma in Asian populations, ${ }^{5-7}$ whereas small series from the United States ${ }^{13}$ and central Europe ${ }^{14}$ showed a much lower incidence (1 and $3 \%$, respectively). Analysis of the prevalence of EBV + diffuse large B-cell lymphoma of the elderly in Latin America is still missing. This prompted us to analyze a series of diffuse large B-cell lymphoma in patients above 50 years from four different hospitals in Mexico City.

The aims of this study were (1) to determine the EBV-association rate in a Mexican cohort of diffuse large B-cell lymphoma of the elderly, (2) to compare it to an analogous cohort from Germany, (3) to determine the EBV strain type and (4) to define the pathological characteristics of the disease.

\section{Patients and methods}

\section{Case Selection}

Cases with the diagnosis of diffuse large B-cell lymphoma, in patients age 50 years and above, from which tissue blocks were available for EBV in situ hybridization, immunohistochemical and molecular analysis were included in the study. From four different Pathology Departments from Mexico City, Mexico, 136 cases were collected. For comparison, 169 cases of diffuse large B-cell lymphoma in patients 50 years or older were selected from the files of the Institute of Pathology, Tübingen, Germany from 2000 to 2009. All cases were reviewed by two pathologists (LQ-M and PA). The medical records were reviewed with special attention to previous medical history.

\section{Immunohistochemical and In Situ Hybridization Analyses}

All cases were subjected to in situ hybridization using oligonucleotides complementary to EpsteinBarr early RNA (EBER) transcripts in paraffin embedded tissue in an automated stainer (Ventana Medical Systems, Tucson, Arizona). EBER positivity in the majority of tumor cells was required to diagnose a case as EBV + diffuse large B-cell lymphoma. A tissue microarray was constructed with the EBV + cases for further immunohistochemical characterization. The paraffin blocks were sampled using $1.0 \mathrm{~mm}$ diameter tissue cores and the cores were arrayed in triplicates. Immunohistochemical analysis was performed on an automated immunostainer (Ventana Medical Systems) according to the company's protocols. The following panel of antibodies was used: BCL-2 (Zytomed Systems, Berlin, Germany), BCL-6 (Zytomed Systems), CD5 (Novocastra, Newcastle, UK), CD10 (Novocastra), CyclinD1 (clone SP6; Epitomics, Burlingame, CA, USA), MUM1 (DAKO Deutschland GmbH, Hamburg, Germany), MIB1 (DAKO), CD20 (clone L26; DAKO), CD79a (DAKO), LMP1 (clone CS.1-4; DAKO), EBNA2 (Novocastra).

\section{Polymerase Chain Reaction Analyses for the Detection of EBV Strain Type and IGH Rearrangements}

DNA used for polymerase chain reaction (PCR) was extracted from $10 \mu \mathrm{m}$ paraffin sections after dewaxing and proteinase $\mathrm{K}$ digestion applying standard phenol/chloroform purification procedures. PCR analysis for EBV A and B strain typing was performed, as previously reported. ${ }^{15}$ Primers flanking a region of the EBNA2 gene differing between type A and type B EBV were used. Each reaction was performed in duplicate with 200 or $500 \eta \mathrm{g}$ of extracted DNA in a $50-\mu$ l volume. The expected fragments for EBV types A and B are 168 and $184 \mathrm{bp}$, respectively. PCR analysis for the $30-\mathrm{bp} L M P 1$ gene 
deletion was performed by using primers flanking the characteristic $30 \mathrm{bp}$ deletion, as previously described, ${ }^{16}$ rendering a 161-bp product for wildtype $L M P 1$ and $131 \mathrm{bp}$ for the deletion variant.

The PCR for IGH gene rearrangements was performed as previously described with BIOMED primers for the framework regions 2 and 3 (FR2 and FR3) using 0.5 U Phusion Hot Start DNA Polymerase (Finnzymes, Woburn, MA, USA) and $200 \mathrm{ng}$ of genomic DNA. ${ }^{17}$ The JH primer was modified with D4 fluorescent dyes (Sigma-Aldrich, St Louis, MO, USA). The products were separated by capillary electrophoresis on the GenomeLab GeXP Genetic Analysis System and analyzed by the GenomeLab GeXP software 10.2 (Beckman Coulter, Brea, CA, USA).

\section{Results}

\section{Patient Characteristics}

EBER positivity was detected by in situ hybridization in the majority of tumor cells in 11 of 136 Mexican cases analyzed. After carefully reviewing the medical records, two patients were excluded from the study because both had a clinical history of rheumatoid arthritis. The remaining nine patients $(7 \%)$ had no history of immunodeficiency, immunosuppressive therapy, prior lymphomas or autoimmune diseases. The clinical data are summarized in Table 1. The group consisted of five males and four females with a median age of 66 years (range 51-75 years). Six patients presented predominantly with lymph node involvement. Three patients presented with extranodal involvement, one in the skin of the face, one with a tumor mass in the tongue and one with spleen involvement. Five patients presented in stage IV disease, one patient in stage III and two patients in stage II. Four patients were lost to follow-up after diagnosis. One patient (case 6) was lost to follow-up with disease in very bad clinical condition, after six cycles of chemotherapy

Table 1 Clinical features of EBV+ DLBCL of the elderly from Mexico

\begin{tabular}{lcccccc}
\hline Case & Sex & Age & Stage & Localization & Treatment & Outcome \\
\hline 1 & F & 58 & IIAe & Face, LN & - & LFU \\
2 & M & 71 & IVB & LN & - & LFU \\
3 & F & 67 & IIIBe & Spleen, LN & CHOPa & DwD (1 month) \\
4 & M & 66 & IVB & LN & MACOP B & DwD (57 months) \\
5 & M & 62 & IVA & LN & CHOP+RT & DwD (22 months) \\
6 & M & 51 & IIA & LN & CHOP & LFU (18 months) \\
7 & F & 71 & IVB & LN & - & LFU \\
8 & F & 60 & IIBe & Tongue, LN & - & DwD $^{\text {c }}(1$ month) \\
9 & M & 75 & IVB & LN & - &
\end{tabular}

AwD: alive with disease; DwD: died with disease; LFU: lost to followup; LN: lymph node; RT: radiotherapy.

${ }^{\mathrm{a}}$ Only one cycle was given.

${ }^{\mathrm{b}}$ The patient was lost to follow-up with disease after six cycles of CHOP.

${ }^{\mathrm{C}}$ The patient died before treatment was started. with cyclophosphamide, doxorubicin, vincristine and prednisone (CHOP); he never achieved complete remission and refused further treatment. Two patients (cases 3 and 4) died within 1 month of the diagnosis. Patient 3 died with disease while receiving the first cycle of chemotherapy. The remaining two patients died with disease 22 and 57 months after diagnosis. The median age of all 136 patients analyzed was 69 years (range 50-92 years).

For comparison, 169 cases of diffuse large B-cell lymphoma in patients above 50 years from Germany were analyzed. EBER positivity was detected in six of 169 cases. Two cases were excluded from the study because of a previous history of marginal zone lymphoma in one patient and a known HIV infection in the second patient. Only four cases fulfilled the criteria of EBV + diffuse large B-cell lymphoma of the elderly, representing $2 \%$ of the German cases, as opposed to the $7 \%$ found in the Mexican cases. The clinical data of these four patients are summarized in Table 2. The patients consisted of two males and two females with a median age of 77 years (range 63-88 years). Three patients presented with stage II and one with stage III. Predominant lymph node involvement was found in three patients, whereas one patient presented with extranodal involvement of the intestine. The latter (case 11) died of sepsis secondary to intestinal perforation before treatment could be started. Two additional patients died with disease (cases 10 and 12). Patient 10 received two cycles of rituximab and bendamustine. This patient had a clinical history of a prostate carcinoma, treated with surgery, hormonal ablation and radiotherapy, 5 years before the lymphoma was diagnosed. Patient 12 received four cycles of CHOP, achieving partial remission; however, 1 month later recurred with massive lymphadenopathy and a tumor mass in the breast. The patient received bendamustine with poor response. Only one patient (case 13) is alive, and in complete remission following R-CHOP therapy 35 months after the primary diagnosis. The average age of all 169 cases analyzed was 72 years (range 51-92 years).

\section{Histological and Immunophenotypic Features}

The histological and immunophenotypic features are summarized in Table 3. Of the nine EBV-positive

Table 2 Clinical features of EBV+ DLBCL from Germany

\begin{tabular}{ccccccc}
\hline \multicolumn{1}{c}{ Case } & Sex & Age Stage Localization & Treatment & Outcome \\
\hline 10 & M & 77 & IIIA & LN & R-Benda & DwD (3 months) \\
11 & F & 88 & IIBe & Intestine, LN & - & DwD \\
12 & F & 77 & IIA & LN & CHOP Benda DwD (11 months) \\
13 & M & 63 & IIA & LN & R-CHOP & CR (35 months)
\end{tabular}

Benda: bendamustine; CR: complete remission; DwD: died with disease; LN: lymph node; R: rituximab; RT: radiotherapy.

${ }^{\mathrm{a}}$ The patient died before treatment was started. 
Table 3 Pathological features of EBV-positive B-cell lymphomas of the elderly

\begin{tabular}{|c|c|c|c|c|c|c|c|c|c|c|c|c|}
\hline Case & Pattern & Necrosis & $C D 20$ & $B C L 2$ & $B C L 6$ & $C D 10$ & MUM1 & $L M P 1$ & EBNA2 & EBV type & $L M P 1 \mathrm{del}$ & IGH clonality \\
\hline \multicolumn{13}{|c|}{ Mexican cases } \\
\hline 1 & Poly & - & + & - & - & - & + & Rare & - & $\mathrm{A}$ & - & ND \\
\hline 2 & Mono & - & + & - & + & + & - & + & - & A & - & Monoclonal \\
\hline 3 & Poly & - & + & $+1-$ & - & - & + & + & - & NA & + & Polyclonal \\
\hline 4 & Poly & +++ & + & + & - & - & + & + & - & B & + & Monoclonal \\
\hline 5 & Poly & - & + & - & - & - & - & + & Rare & A & - & ND \\
\hline 6 & Poly & - & + & - & - & - & + & + & - & A & - & Monoclonal \\
\hline 7 & Poly & - & + & - & - & - & + & + & - & B & + & Monoclonal \\
\hline 8 & Poly & +++ & + & - & + & - & + & + & - & B & + & Polyclonal \\
\hline 9 & Poly & +++ & + & - & + & - & + & + & - & B & + & Monoclonal \\
\hline \multicolumn{13}{|c|}{ German cases } \\
\hline 10 & Poly & - & + & + & - & - & + & + & + & B & - & Monoclonal \\
\hline 11 & Mono & - & $+/-^{a}$ & $+1-$ & + & - & + & + & - & A & - & Monoclonal \\
\hline 12 & Mono & - & + & + & - & + & - & + & - & A & + & Monoclonal \\
\hline 13 & Poly & - & + & - & - & - & + & - & - & A & - & Polyclonal \\
\hline
\end{tabular}

Mono: monomorphic type; NA: not assigned; ND: not done; Poly: polymorphic type.

${ }^{\mathrm{a}}$ This case was mainly CD79 a positive.

diffuse large B-cell lymphomas identified in Mexican patients, eight revealed a polymorphous infiltrate characterized by a mixture of small lymphocytes, histiocytes, plasma cells and lymphoid cells with plasmacytic differentiation, immunoblasts and large atypical lymphoid cells and Reed-Sternberg-like cells (Figure 1a and b). In three cases (33\%) (cases 4, 8 and 9), geographic areas of necrosis with apoptotic tumor cells, surrounded by EBER + cells, were observed (Figure 1c and d). All polymorphic cases expressed CD20 and CD79a (Figure 1e), and showed a non-germinal center B-cell phenotype characterized by CD10 negativity and in general MUM1 positivity (Figure 1f). BCL-2 was generally negative. The large Reed-Sternberglike cells were also CD20 positive, usually CD30 positive (Figure 1g) and lacked CD15. Only one case (case 2) showed a monomorphic morphology with sheets of centroblasts (Figure 2a). Almost $100 \%$ of tumor cells were EBER + (Figure 2b). Phenotypically, the tumor cells revealed a germinal center B-cell phenotype (CD20+, CD10 + and BCL-6+) (Figure 2c-e). LMP1 was positive in all cases (Figure 2f), whereas EBNA2 was positive in rare cells in only one case $(11 \%)$ (case 5 ).

Of the four EBV + German cases, two had a polymorphic and two a monomorphic cytology. All cases were CD20 and CD79a positive. Case 10, a polymorphic type showed a non-germinal center B-cell phenotype (BCL-2 +, CD10-, MUM1+) and was positive for LMP1 and EBNA2 (Figure 1h). Case 13, a polymorphic type was unusual in that LMP1 and EBNA2 were both negative and that the patient achieved complete remission.

Morphologically and phenotypically the Mexican and German cases were very similar. Of the 13 cases combined, $10(77 \%)$ were of the polymorphic type and $3(23 \%)$ were monomorphic. Twelve (92\%) were LMP1 positive and two (15\%) were EBNA2 positive.
Interestingly, seven additional samples of the 136 Mexican DLBCL (5\%) showed EBER positivity in $<20 \%$ of cells (Figure 3). The patients were five females and two males with a median age of 70.5 years (range 56-87 years). These cases were not regarded as EBV + diffuse large B-cell lymphoma of the elderly. In contrast to the Mexican cases, only one German case showed partial EBER positivity. ( $5 \%$ vs $0.5 \%$, respectively).

\section{EBV Genotype and Clonality Analyses}

Of the nine Mexican cases analyzed (lanes 1-9), four of the eight evaluable cases were EBV strain type A, and four cases type B EBV (Figure 4). The 30-bp del$L M P 1$ variant was identified in five of the nine cases including the four cases with type B EBV. All four type A EBV carried the wild-type $L M P 1$ gene. Of the four German cases, three were EBV type A (lanes 11-13) and one was EBV type B (lane 10). One EBV type A case carried the LMP1 $30 \mathrm{bp}$ deletion (lane 12). The results of clonality analysis are summarized in Table 3. Of the 11 evaluable cases, 8 showed a B-cell monoclonal population and three were polyclonal.

\section{Discussion}

In this study, we analyzed a series of diffuse large B-cell lymphoma in patients aged 50 years or greater for the presence of EBV infection by in situ hybridization in a Mexican population, and compared it with a German population. The prevalence of EBV + diffuse large B-cell lymphoma of the elderly in the Mexican patients was significantly higher than in the German cohort ( $7 \%$ vs $2 \%$ ), and the lymphomas occurred approximately one decade earlier (66 and 77 years, respectively). The preva- 

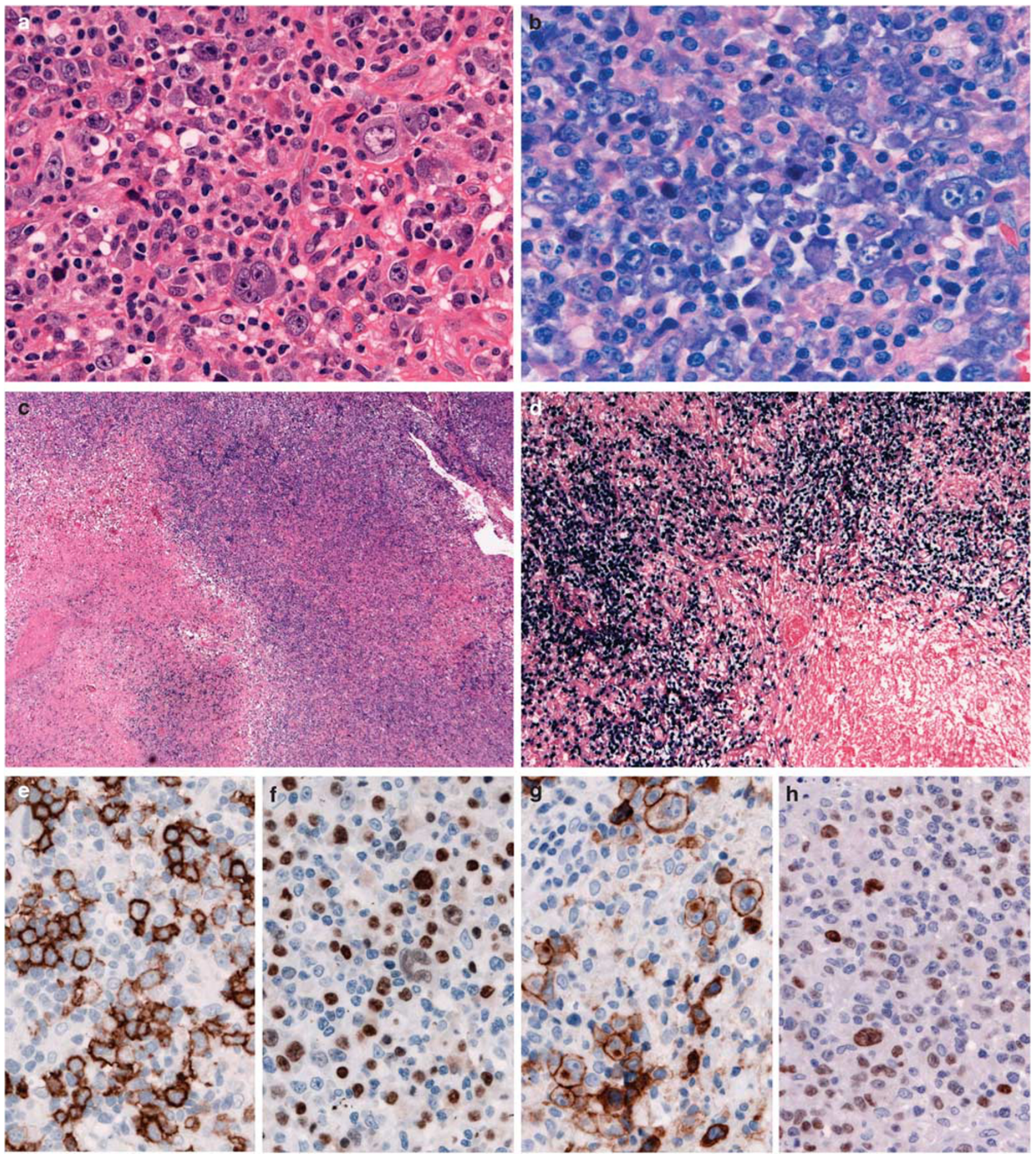

Figure 1 Polymorphic type of EBV-positive B-cell lymphoma of the elderly. (a) A polymorphous proliferation characterized by small lymphocytes, plasmacytic and plasmablastic cells, and scattered large transformed cells mimicking Hodgkin and Reed-Sternberg cells is observed (hematoxylin-eosin; original magnification $\times 400$ ). (b) Giemsa stain highlights the plasmacytic differentiation of the tumor cells. (Giemsa stain; original magnification $\times 400$ ). (c) Low magnification of a lymph node diffusely infiltrate with large geographic areas of necrosis (hematoxylin-eosin; original magnification $\times 50$ ). (d) EBER in situ hybridization shows diffuse EBV-positive tumor cells surrounding the necrosis (original magnification $\times 100$ ). $(\mathbf{e})$ CD20 stain highlights the large, transformed cells (original magnification $\times 400$ ). (f) Note that most of the tumor cells are positive for MUM1 (original magnification $\times 400$ ). (g) CD30 stain is positive in most of the large transformed Hodgkin and Reed-Sternberg-like cells (original magnification $\times 400$ ). (h) Case 10. EBNA 2 stain is positive in many small and large tumor cells (original magnification $\times 400$ ).

lence of EBV + diffuse large B-cell lymphoma of the elderly in Mexican patients is comparable with the data published in the Asian literature
(7\% vs $8-10 \%))^{5-7}$ However, the median age at presentation in Mexican patients was relatively low (66 years) when compared with the Japanese series 



Figure 2 Monomorphic type of EBV-positive B-cell lymphoma of the elderly (case 2). (a) The lymph node is diffusely replaced by a monomorphic infiltrate of large centroblastic cells with abundant mitosis (hematoxylin-eosin; original magnification $\times 400)$. (b) EBER in situ hybridization shows diffuse EBV-positive tumor cells. Note that almost $100 \%$ of the tumor cells are EBER positive (original magnification $\times 100)$. (c) CD20 stain is positive in the large tumor cells (original magnification $\times 400$ ). (d) The tumor cells are strongly positive for CD10 (original magnification $\times 400$ ). (e) BCL-6 is also positive in the tumor cells (original magnification $\times 400$ ). (f) LMP1 stain shows specific cytoplasmic positivity in a minority of the tumor cells (original magnification $\times 400$ ).

(71 years). ${ }^{5,6}$ An interesting finding was that an additional $5 \%$ percent of the diffuse large B-cell lymphoma in Mexican population showed EBV positivity in $<20 \%$ of the large cells. Although these cases did not fulfill the criteria of EBV + diffuse large B-cell lymphoma of the elderly, it suggests that in rare cases, secondary EBV infection of an established B-cell clone can occur. ${ }^{18}$ A similar scenario has been reported in a T-cell lymphoma. ${ }^{19}$ Alternatively, these cells could represent clonally 

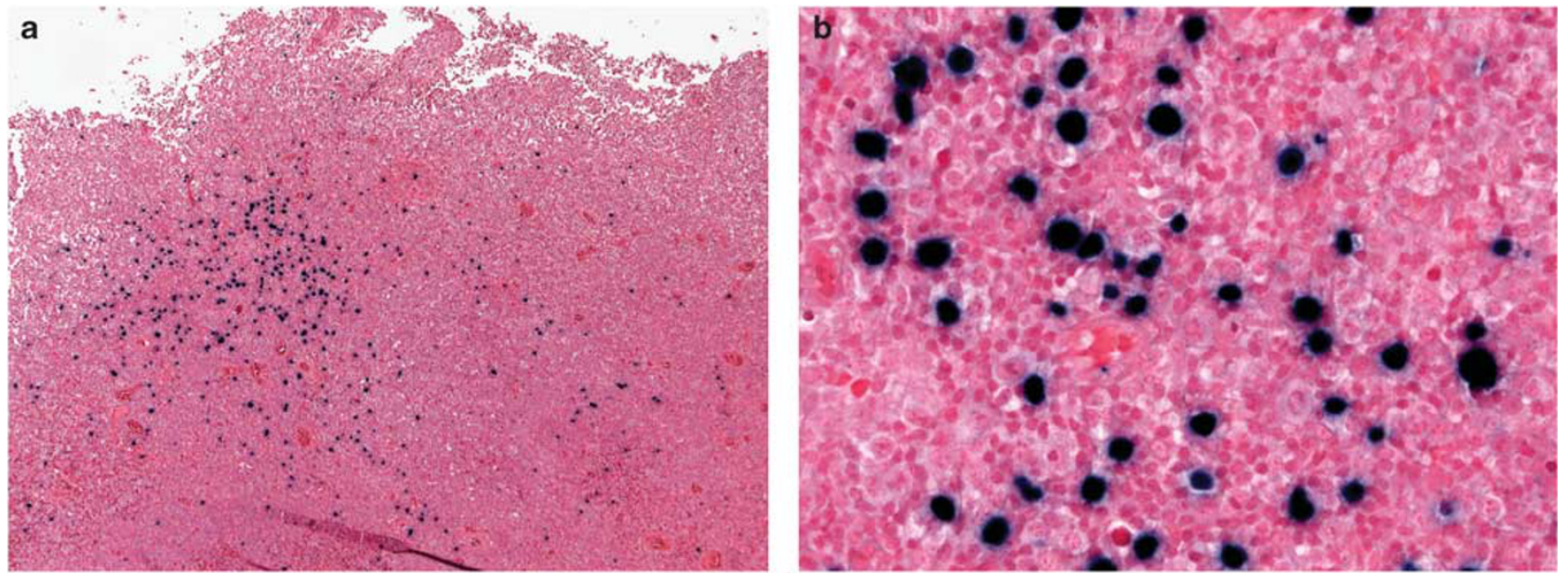

Figure 3 Partial EBV positivity in diffuse large B-cell lymphomas. (a) EBER in situ hybridization shows focal EBV-positive tumor cells (original magnification $\times 50$ ). (b) At higher magnification, the large positive tumor cells and small lymphocytes are depicted (original magnification $\times 400)$.

\section{a}

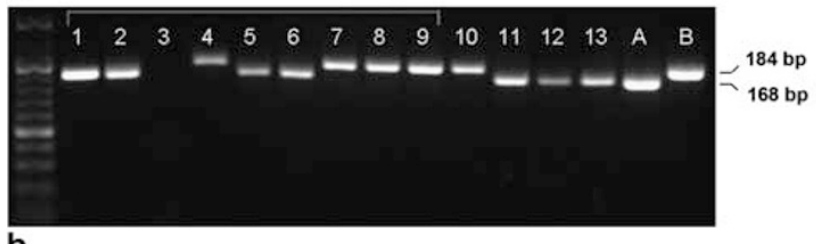

b

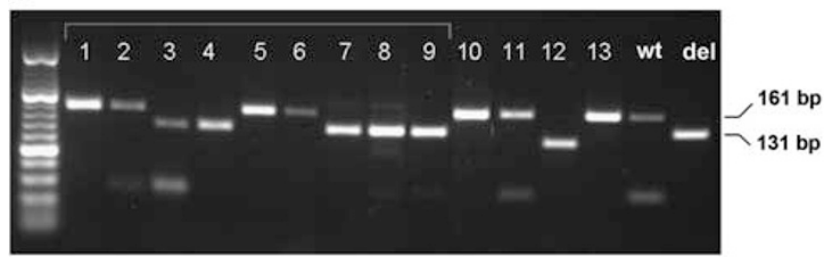

Figure 4 (a) EBV subtyping by PCR analysis from EBNA2 gene sequences. The primers generate a specific $168 \mathrm{bp}$ fragment for specimens containing type A EBV, and a specific $184 \mathrm{bp}$ fragment for samples infected by type B EBV. Lanes 1-9 represent the Mexican cases, lanes 10-13 the German cases. (a, b) are the positive controls. (b) PCR analysis of the 30-bp LMP1 gene deletion. The 161-bp fragment represents the full-length (wildtype) $L M P 1$ gene configuration, whereas the 131-bp fragment represents the 30-bp LMP1 deletion. Lanes 1-9 represent the Mexican cases, lanes 10-13 the German cases. wt, wild-type control and del, LMP1 $30 \mathrm{bp}$ deletion control.

unrelated EBV transformed B cells and might reflect the higher incidence of and/or susceptibility for EBV infection in Mexican population. The prevalence of $2 \%$ found in the German cohort corroborates previous data published from central Europe. ${ }^{14}$ Furthermore, in a small series from the Midwestern USA, the overall rate of EBV positivity in otherwise typical diffuse large B-cell lymphoma in older adults was approximately $1 \% \cdot{ }^{13}$ The published studies until now indicate a lower prevalence of EBV + diffuse large B-cell lymphoma of the elderly in Western populations, as compared with Asians and the Mexican population analyzed in this study. These results are not that unexpected, since other EBV-associated disorders have been shown to be more prevalent in Asian and Latin American populations.

In the original description of EBV + diffuse large B-cell lymphoma of the elderly, the frequent extranodal presentation was emphasized as a hallmark feature of this disease (up to $80 \%$ ). ${ }^{5}$ Nevertheless, in an extended study from the same Japanese group, ${ }^{6}$ the authors described a high but similar frequency of extranodal involvement in elderly patients with both EBV-positive and EBV-negative DLBCL. In our study, only $30 \%$ of the cases showed extranodal involvement; however, all of them had lymph node involvement at the time of diagnosis. Accordingly, most recent series have found a predominantly nodal involvement in EBV + diffuse large B-cell lymphoma of the elderly. ${ }^{13,14,20}$

Although the mechanism underlying the development of these lymphomas is not understood, it is believed that these lesions arise secondary to deterioration of the immune system due to the aging process. Probably, the best evidence that acquired immunodepression might have an etio-pathogenic role is the fact that around $30 \%$ of EBV + diffuse large B-cell lymphoma of the elderly reported from Asia express EBNA2, and, therefore, show a latency III EBV infection, typical of immunodeficiencyassociated lymphomas. Of note, 4 of the original $17 \mathrm{EBV}+$ cases, all of them EBNA2 +, had to be excluded from our study because after carefully reviewing the medical records, a cause for immunosuppression or immune dysregulation was identified (autoimmune disease, two cases; previous lymphoma one case, HIV positivity, one case). Moreover, one of the remaining two cases with EBNA2 expression (case 10) had a history of prostate carcinoma treated with surgery, hormonal ablation and radiotherapy 5 years prior to development of the $\mathrm{EBV}+$ diffuse large B-cell lymphoma of the elderly. Although previous history of carcinoma does not preclude the diagnosis of EBV + diffuse large B-cell lymphoma of the elderly, it is tempting to speculate 
that this fact influenced the development of the EBV + diffuse large B-cell lymphoma of the elderly in this patient. Furthermore, besides the EBNA2 positivity, this was the only case with type B EBV without $L M P 1$ deletion. Type B EBV without $L M P 1$ deletion can only effectively transform B cells in immunodeficient patients, as has been shown in HIV + patients. ${ }^{16,21,22}$ This evidence strongly suggests that this patient might have had a more profound immunosuppression than the presumed age-related immune dysfunction. In our experience, EBNA2 expression in EBV + diffuse large B-cell lymphoma of the elderly is rather rare. We strongly believe that patients with EBV + diffuse large B-cell lymphoma with EBNA2 expression should be studied carefully for an underlying cause of immunosuppression.

Another point of interest is the high frequency of type B EBV with the LMP1 $30 \mathrm{bp}$ deletion in the Mexican cohort (50\%, four of eight cases). These results corroborate our previous findings in classical Hodgkin lymphoma in a Mexican population without obvious immunodeficiency. ${ }^{23}$ This finding most probably reflects the geographic variation in EBV strain type rather than differences in the pathogenesis of the disease or the immune status of the host.

Morphologically, the cases analyzed in this study did not differ from previous reports. ${ }^{5,6,13,14}$ Most of the cases corresponded to the polymorphic variant of EBV + diffuse large B-cell lymphoma of the elderly (10 of 13 cases). The tumor cells were in all instances CD20 positive, often CD30 positive, and lack CD15 expression. The strong, homogeneous CD20 positivity combined with the lack of CD15 are useful features in the differential diagnosis with EBV + classical Hodgkin lymphoma. ${ }^{24}$ As already described, ${ }^{13}$ all polymorphic cases revealed a nongerminal center B-cell phenotype. In contrast, two of the three EBV + diffuse large B-cell lymphoma of the elderly with monomorphic type showed a germinal center B-cell phenotype with expression of CD10 and BCL-6 or BCL-2. As reported in the literature, the majority of our cases were monoclonal, as demonstrated by IGH PCR (8 of 11 cases).

In conclusion, we found a similar prevalence of $\mathrm{EBV}+$ diffuse large B-cell lymphoma of the elderly in a Mexican population compared with what has been reported from Asian countries, and in contrast to the low frequency in Western populations. However, compared with the Asian series, the Mexican patients were younger at diagnosis, presented predominantly with nodal disease and rarely expressed EBNA2. An interesting finding was the high frequency of EBV type B with $L M P-1$ deletion found in the Mexican cases.

\section{Acknowledgement}

We thank Claudia Kloss and Anne Adam for their valuable technical assistance with the immuno- histochemistry and in situ hybridization analyses and Sema Colak for her help in the molecular analysis of the specimens.

\section{Disclosure/conflict of interest}

The authors declare no conflict of interest.

\section{References}

1 Cohen JI. Epstein-Barr virus infection. N Engl J Med 2000;343:481-492.

2 Gottschalk S, Rooney CM, Heslop HE. Post-transplant lymphoproliferative disorders. Annu Rev Med 2005;56:29-44.

3 Cohen JI, Kimura H, Nakamura S, et al. Epstein-Barr virus-associated lymphoproliferative disease in nonimmunocompromised hosts: a status report and summary of an international meeting, 8-9 September 2008. Ann Oncol 2009;20:1472-1482.

4 Cohen JI, Bollard CM, Khanna R, et al. Current understanding of the role of Epstein-Barr virus in lymphomagenesis and therapeutic approaches to EBV-associated lymphomas. Leuk Lymphoma 2008;49(Suppl 1):27-34.

5 Oyama T, Ichimura K, Suzuki R, et al. Senile EBV+ Bcell lymphoproliferative disorders: a clinicopathologic study of 22 patients. Am J Surg Pathol 2003;27:16-26.

6 Oyama T, Yamamoto K, Asano N, et al. Age-related EBV-associated B-cell lymphoproliferative disorders constitute a distinct clinicopathologic group: a study of 96 patients. Clin Cancer Res 2007;13:5124-5132.

7 Park S, Lee J, Ko YH, et al. The impact of Epstein-Barr virus status on clinical outcome in diffuse large B-cell lymphoma. Blood 2007;110:972-978.

8 Nakamura S, Jaffe ES, Swerdlow SH. EBV positive diffuse large B-cell lymphoma of the elderly. In: Swerdlow SH, Campo E, Harris NL, Jaffe ES, Pileri S, Stein $\mathrm{H}$, et al. (eds). WHO Classification of Tumours of Haematopoietic and Lymphoid Tissues, 4th edn. International Agency for Research on Cancer: Lyon, 2008;243-244.

9 Elenitoba-Johnson KS, Zarate-Osorno A, Meneses A, et al. Cytotoxic granular protein expression, EpsteinBarr virus strain type, and latent membrane protein-1 oncogene deletions in nasal T-lymphocyte/natural killer cell lymphomas from Mexico. Mod Pathol 1998;11:754-761.

10 Quintanilla-Martinez L, Kumar S, Fend F, et al. Fulminant EBV(+) T-cell lymphoproliferative disorder following acute/chronic EBV infection: a distinct clinicopathologic syndrome. Blood 2000;96:443-451.

11 Quintanilla-Martinez L, Gamboa-Domnquez A, Gamez-Ledesma I, et al. Association of Epstein-Barr virus latent membrane protein and Hodgkin's disease in Mexico. Mod Pathol 1995;8:675-679.

12 Gulley ML, Eagan PA, Quintanilla-Martinez L, et al. Epstein-Barr virus DNA is abundant and monoclonal in the Reed-Sternberg cells of Hodgkin's disease: association with mixed cellularity subtype and Hispanic American ethnicity. Blood 1994;83: 1595-1602.

13 Gibson SE, Hsi ED. Epstein-Barr virus-positive B-cell lymphoma of the elderly at a United States tertiary 
medical center: an uncommon aggressive lymphoma with a nongerminal center B-cell phenotype. Hum Pathol 2009;40:653-661.

14 Hoeller S, Tzankov A, Pileri SA, et al. Epstein-Barr virus-positive diffuse large B-cell lymphoma in elderly patients is rare in western populations. Hum Pathol 2010;41:352-357.

15 Quintanilla-Martinez L, Lome-Maldonado C, Schwarzmann F, et al. Post-transplantation lymphoproliferative disorders in Mexico: an aggressive clonal disease associated with Epstein-Barr virus type A. Mod Pathol 1998;11:200-208.

16 Kingma DW, Weiss WB, Jaffe ES, et al. Epstein-Barr virus latent membrane protein-1 oncogene deletions: correlations with malignancy in Epstein-Barr virusassociated lymphoproliferative disorders and malignant lymphomas. Blood 1996;88:242-251.

17 van Dongen JJ, Langerak AW, Bruggemann $\mathrm{M}$, et al. Design and standardization of PCR primers and protocols for detection of clonal immunoglobulin and T-cell receptor gene recombinations in suspect lymphoproliferations: report of the BIOMED-2 concerted action BMH4-CT98-3936. Leukemia 2003;17: 2257-2317.

18 Quintanilla-Martinez L, Lome-Maldonado C, Ott G, et al. Primary non-Hodgkin's lymphoma of the intestine: high prevalence of Epstein-Barr virus in Mexican lymphomas as compared with European cases. Blood 1997;89:644-651.
19 Langer R, Geissinger E, Rudiger T, et al. Peripheral T-cell lymphoma with progression to a clonally related, Epstein Barr virus+, cytotoxic aggressive T-cell lymphoma: evidence for secondary EBV infection of an established malignant T-cell clone. Am J Surg Pathol 2010;34:1382-1387.

20 Quintanilla-Martinez L, de Jong D, de Mascarel A, et al. Gray zones around diffuse large B cell lymphoma. Conclusions based on the workshop of the XIV meeting of the European Association for Hematopathology and the Society of Hematopathology in Bordeaux, France. J Hematop 2009;2:211-236.

21 Young LS, Rickinson AB. Epstein-Barr virus: 40 years on. Nat Rev Cancer 2004;4:757-768.

22 Chang CM, Yu KJ, Mbulaiteye SM, et al. The extent of genetic diversity of Epstein-Barr virus and its geographic and disease patterns: a need for reappraisal. Virus Res 2009;143:209-221.

23 Dirnhofer S, Angeles-Angeles A, Ortiz-Hidalgo C, et al. High prevalence of a 30 -base pair deletion in the Epstein-Barr virus (EBV) latent membrane protein 1 gene and of strain type B EBV in Mexican classical Hodgkin's disease and reactive lymphoid tissue. Hum Pathol 1999;30:781-787.

24 Asano N, Yamamoto K, Tamaru J, et al. Age-related Epstein-Barr virus (EBV)-associated B-cell lymphoproliferative disorders: comparison with EBV-positive classic Hodgkin lymphoma in elderly patients. Blood 2009;113:2629-2636. 